Rev. Biol. Trop., 47(4): 851-865, 1999

www.ucr.ac.cr www.ots.ac.cr www.ots.duke.edu

\title{
Estructura y variación estacional de poblaciones de moluscos asociadas a la pesca artesanal de langosta en el Pacífico Tropical
}

\section{Celia Olabarria}

Departamento de Invertebrados y Ecología del Bentos, Facultad de Ciencias del Mar, Universidad Autónoma de Sinaloa, C/Paseo Claussen, s/n. 82000 Mazatlán, Sinaloa, México. Fax: (69)828656. e-mail: chispita@ola.icmyl.unam.mx

\author{
Recibido 10-I-1999. Corregido 21-VI-1999. Aceptado 24-VI-1999
}

\begin{abstract}
Mollusks associated to the spiny lobster fishery during 1997-1998 in the Tropical Pacific were surveyed. A total of 1081 specimens of 11 species were collected. The greatest captures were in autumn 1997 (28.16\%) and summer 1998 (25.58\%). Dominant species were Hexaplexnigritus (Philippi 1845) (83.10\%), Chicoreus regius (Swainson 1821) (8.04\%), Vasum caestus (Broderip 1833) (2.12\%), C. brassica (Lamarck 1822) (1.85\%) and C. erythrostomus (Swainson 1831) (1.75\%), which have commercial importance. Weight-height ratio, sex ratio and height frequency histograms were done for dominant species. Sex ratio was 1:1, except for $C$. erythrostomus and H. nigritus (2 males: 1 female); C. regius, C. erythrostomus and V. caestus presented an allometric growth while $H$. nigritus and $C$. brassica grew isometrically. Only $H$. nigritus males were larger than conspecific females. There were significative differences in species distribution in relation with depth; $C$. erythrostomus occurred in deepest layers while $H$. nigritus was more superficial; $H$. nigritus size differed with depth, temperature and locality.
\end{abstract}

Key words: Mollusks, diversity, distribution, weight-height ratio, population structure, lobster fishery, Sinaloa, Mexico.

\section{INTRODUCCIÓN}

En el sureste del Golfo de California habitan dos especies de langosta, Panulirus inflatus (Bouvier 1895) y P. gracilis Streets 1871. Su captura constituye una significativa fuente de ingreso para los pescadores (a nivel local o regional) debido a su alta cotización en el mercado (Flores-Campaña et al. 1993). En Mazatlán se capturan estas dos especies de langosta, explotándose de manera diferente y no tradicional, utilizando la red de enmalle o "chinchorro". Este tipo de arte de pesca captura, junto con la langosta, otras especies de interés comercial: peces, otros crustáceos y moluscos (Pérez-González et al. 1994). Esta fauna aso- ciada a la pesca de la langosta (FAL) es una opción importante de ingresos para los pescadores, sobre todo en algunas épocas del año cuando las capturas de langosta son bajas.

Los trabajos de la fauna asociada a la pesca de recursos de interés comercial en las costas del sur de Sinaloa son escasos, destacando los realizados sobre fauna asociada al camarón (Hendrickx et al. 1984a,b, Hendrickx 1985, 1990), y los referentes a la fauna asociada a la pesca artesanal de la langosta (Hendrickx et al. 1992, 1997, Pérez-González et al. 1994, Ayón et al. 1997, van der Heiden \& Plasencia-González 1997).

En lo que respecta a moluscos, es poca la información disponible sobre pesquerías potenciales en la plataforma continental del sureste 
de Sinaloa, destacando los trabajos llevados a cabo por Rodríguez de la Cruz (1981), van der Heiden \& Hendrickx (1982) y Hendrickx et al. (1984b). Debido a la importancia de los moluscos como recurso potencial, y a la ausencia de trabajos de esta naturaleza en este área, decidimos hacer un estudio orientado a conocer la variedad y estructura de las poblaciones de moluscos asociados a la pesca artesanal de langosta durante el período comprendido entre octubre de 1997 y octubre de 1998, época que coincidió con el desarrollo de el fenómeno de El Niño. Asimismo, analizamos las variaciones estacionales que existieron entre la proporción de biomasa de langosta y la de moluscos, y las relaciones entre la distribución y tamaño de las distintas especies, y algunos factores abióticos (temperatura y profundidad).

\section{MATERIALES Y MÉTODOS}

Área de estudio: La zona de estudio se localiza en la costa sur de Sinaloa (México) entre los $23^{\circ} 10^{\prime} 05^{\prime \prime}$ y $23^{\circ} 29^{\prime} 01^{\prime \prime} \mathrm{N}$ y los $106^{\circ} 24^{\prime} 09^{\prime \prime}$ y $106^{\circ} 40^{\prime} 05^{\prime \prime} \mathrm{W}$. El área de muestreo abarcó una profundidad comprendida entre 5 y $38 \mathrm{~m}$, predominando los fondos arenosos que en algunos casos presentaron manchas de sustrato rocoso.

La región presenta un clima cálido húmedo con lluvias en verano, siendo el más seco de los subhúmedos, con régimen de lluvias en verano. La temperatura media anual es de $27^{\circ} \mathrm{C}$ y la precipitación promedio de $502.8 \mathrm{~mm}$ (García 1973). Los vientos dominantes son del oeste y noroeste, con una velocidad promedio de 2.6 a $3.5 \mathrm{~m} / \mathrm{s}$.

Las olas más fuertes provienen del noroeste y del norte en período de invierno y, generalmente producen una deriva litoral hacia el sur. Durante el régimen de verano el oleaje que proviene del sur provoca una deriva litoral hacia el norte (Juárez-Rosales 1995). La zona se ve afectada por tormentas tropicales y huracanes que se forman en el $\mathrm{Pa}$ cífico nororiental, observándose una mayor incidencia de éstos en los meses comprendidos entre julio y octubre.
El período de muestreo coincidió con la aparición de El Niño 98. Este fenómeno ocurre cuando los vientos alisios del este disminuyen, entonces una corriente cálida de agua, a una profundidad de $150 \mathrm{~m}$, fluye sobre las aguas más frías desde el Oceano Pacífico ecuatorial hacia el este. Esto origina que el agua templada se mueva hacia la costa, y se producen cambios significativos en el clima. El Niño ocasiona fenómenos fuertes que alteran la temperatura de los ecosistemas en el Hemisferio Norte, originando altas temperaturas no usuales, especialmente durante el invierno (Suplee 1999).

Recolección de muestras: Se realizaron dos muestreos por mes desde octubre de 1997 a octubre de 1998, utilizando las embarcaciones de los pescadores (madera revestidas de fibra de vidrio, con motores fuera borda de 25,48 o $75 \mathrm{HP}$ ). Las muestras fueron recolectadas con el equipo empleado en la pesca de la langosta (redes de enmalle de seda o nilón, con una longitud de 75 a $200 \mathrm{~m}$, caída de $2.60 \mathrm{~m}$, y luz de malla de 8.75 a $15 \mathrm{~cm}$ ). Los parámetros físicos que se midieron fueron la temperatura del agua de mar (superficial y de fondo) y la profundidad. En este trabajo también se analizaron las temperaturas superficiales del agua en tres años anteriores, con el objeto de mostrar los efectos de El Niño 1998. Los datos de temperatura que corresponden a la media mensual a lo largo del año fueron aportados por el Centro Regional de Investigación Pesquera de Mazatlán. La temperatura se midió con un termómetro de cubeta graduado de $-1{ }^{\circ} \mathrm{C}$ a $51{ }^{\circ} \mathrm{C}\left( \pm 0.1{ }^{\circ} \mathrm{C}\right)$ y la profundidad se obtuvo con una sondaleza.

Los organismos capturados fueron cuantificados, identificados y preservados. En todos los casos fue extraída la parte blanda del animal para proceder a la determinación del sexo, posteriormente se fijó al $10 \%$ y se guardó en alcohol al 70\%. Los datos merísticos registrados fueron la altura (desde el ápice hasta el extremo inferior de la abertura), anchura de la concha (en la espira del cuerpo, justo encima de la abertura), y el peso total. Estas medidas fueron tomadas utilizando un vernier con precisión de $\pm 0.1 \mathrm{~mm}$ y una balanza de precisión $\pm 0.1 \mathrm{~g}$, respectivamente. 
Análisis de datos: Se utilizaron los índices univariantes de diversidad de Shannon (Shannon-Weaver 1963), equitatividad (Pielou 1966) y riqueza de especies (Margalef 1974) para analizar los datos biológicos en las distintas localidades y épocas de muestreo, usando logaritmo en base 2 .

La similitud entre las localidades y épocas del período de muestreo fue analizada por medio de técnicas multivariantes de ordenación, previa transformación de los datos usando la raíz cuarta, cuyo objeto es reducir el peso de las especies más abundantes (Field et al. 1982). La matriz de similitud para el análisis de ordenación se calculó por medio del índice Bray-Curtis (Bray \& Curtis 1957). El análisis de ordenación utilizado fue el MDS (Non-metric multidimensional scaling program), determinándose el nivel de significación del análisis por el coeficiente de estrés de Kruskal (Clarke 1993). Para determinar las especies más comunes se utilizó el índice de constancia de Dajoz (1971), que se define según la siguiente ecuación:

$$
C_{A 1}=\frac{N_{A 1}}{N_{1}} \times 100
$$

donde la constancia de una especie $\mathrm{A}\left(\mathrm{C}_{\mathrm{A} 1}\right)$ en una comunidad o muestra, es la relación entre el número de muestreos donde existe la especie $\left(\mathrm{N}_{\mathrm{A} 1}\right)$ y el número de muestras dentro de esa comunidad $\left(\mathrm{N}_{1}\right)$.

Las desviaciones de la relación de sexos $1: 1$ se estimaron mediante la prueba $\mathrm{X}^{2}$ de bondad de ajuste (Snedecor \& Cochran 1980). Los datos biométricos de las cinco especies más comunes se utilizaron para realizar histogramas de frecuencia de tallas y conocer la relación talla-peso. Esta se expresa según Ricker (1975) como una función potencial según la ecuación:

$$
P=a X^{b}
$$

donde, P: peso total (g), X: longitud (mm), b: pendiente, a: ordenada al origen.

Con los datos de pesos registrados para langosta y moluscos durante este estudio, se estimó la proporción que representaron los moluscos por estaciones del año, en total y por especies.
Posteriormente, se analizó la relación entre la distribución del número de individuos, y los parámetros temperatura y profundidad, mediante el coeficiente de correlación de rango de Spearman. Además, para cada una de las especies dominantes se analizó la relación entre la talla y los parámetros físicos (temperatura y profundidad), y la variación de tallas entre sexos, utilizando el coeficiente de correlación de rango de Spearman y análisis de Kruskal-Wallis (Sokal \& Rohlf 1979), respectivamente.

\section{RESULTADOS}

El área de estudio presentó una temperatura superficial media del agua de $26.89^{\circ} \mathrm{C}$, con una mínima de $23{ }^{\circ} \mathrm{C}$ en abril de 1998 y una temperatura máxima de $30.8^{\circ} \mathrm{C}$ en agosto de 1998. La temperatura de fondo siguió una variación paralela a la temperatura superficial, registrándose la más alta en el mes de agosto $1998\left(29.9^{\circ} \mathrm{C}\right)$ y la mínima en abril de 1998 $\left(22.5^{\circ} \mathrm{C}\right)$, con una temperatura media durante del período de estudio de $26.3{ }^{\circ} \mathrm{C}$ (Fig.1). Al analizar los datos de los dos años anteriores, se pudo observar como las temperaturas empezaron a elevarse a finales de 1997 cuando comenzó El Niño 98, alcanzando el máximo desarrollo durante el invierno de 1998, causando unas temperaturas medias mucho más altas que en años anteriores $\left(>4-5^{\circ} \mathrm{C}\right)$. Al final del año 1998, sin embargo, la temperatura media del agua fue mucho más baja, marcándose el comienzo de La Niña.

A bundancia, diversidad y similitud entre las épocas y localidades de muestreo: Se recolectó un total de 1081 individuos pertenecientes a cinco familias, ocho géneros y 11 especies. De las 11 especies, la Familia Muricidae fue la mejor representada con 3 géneros y 6 especies (54.5\% de las especies). La Familia Buccinidae representó el segundo lugar con 2 géneros y 2 especies (18.2\%). Las Familias Tonnidae, Vasidae y Conidae, estuvieron representadas por un género y una especie, lo que supuso el $9.1 \%$ del total de las especies. 


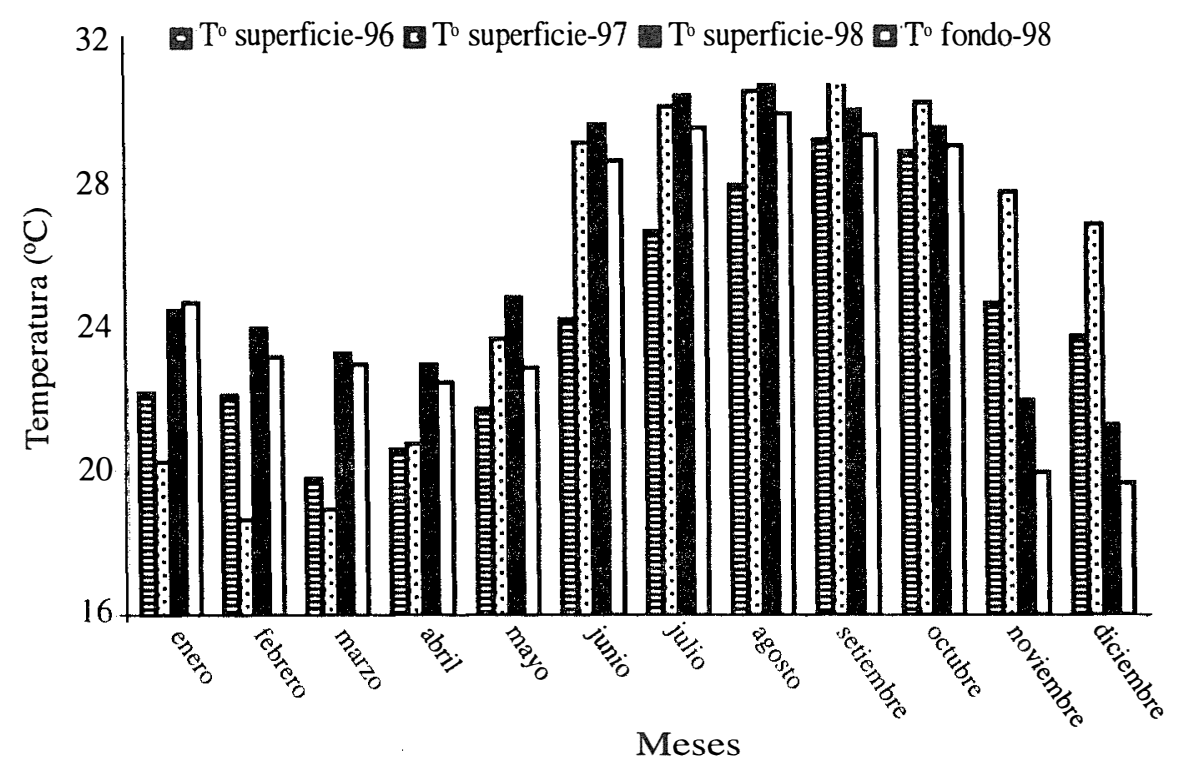

Fig. 1. Distribución de la temperatura $\left({ }^{\circ} \mathrm{C}\right)$ de superficie desde 1996 hasta 1998 en el sur de Sinaloa. También aparece reflejada la temperatura de fondo durante 1998.

Fig. 1. Distribution of surface temperature from 1996 to 1998 in the southern Sinaloa. Also, bottom temperature during 1998 is showed.

Por localidades de muestreo, la mayor abundancia se registró en las estaciones E6 y E8 (estaciones alejadas de Mazatlán), observándose una disminución del número de individuos en las localidades más próximas a Mazatlán. Por épocas, la mayor abundancia se registró en otoño de 1997, mientras que la más baja se dio en invierno de 1998. En primavera de 1998 y otoño de 1998 las abundancias fueron similares, registrándose un aumento considerable de las capturas en verano de 1998 (Cuadro 1). Al considerar la abundancia numérica de cada especie se observó que Hexaplex nigritus (Philippi 1845) fue la especie más abundante (83.10\%), seguida de Chicoreus regius (Swainson 1821) con el $8.04 \%$, y.Vasum caestus (Broderip 1833) con 2.12\%. C. brassica (Lamarck 1822), C. erythrostomus (Swainson 1831), Malea ringens (Swainson 1822) y Pleuropoca princeps (Sowerby 1825) presentaron una abundancia mucho menor que representó el $1.85 \%, 1.75 \%, 1,11 \%$ y $1.11 \%$ del total de individuos, respectivamente. Homalocantha oxyacantha (Broderip 1833), Cantharus gemmatus (Reeve 1846), Conus fergusoni
Sowerby 1873 y $H$. princeps (Broderip 1833) tuvieron las menores abundancias $(0.37 \%$, $0.18 \%, 0.18 \%$ y $0.10 \%$, respectivamente): Las siete primeras especies son comestibles, siendo la primera especie la que presenta un mayor valor comercial.

Los índices de diversidad fueron bajos (inferiores a 1) debido a la dominancia de $H$. $n i$ gritus en todas las localidades y épocas de muestreo. El análisis de localidades mostró la mayor diversidad en E7 (0.65) y E9 (0.59), y la menor en E4 (0.05). Asimismo, la equitatividad alcanzó su máximo valor en E7 (0.84), mientras que el mínimo fue en E4 (0.10). Los valores bajos de diversidad se debieron más a un aumento de la dominancia de $H$. nigritus que a una disminución en el número de especies. Sin embargo, la riqueza mostró un comportamiento muy diferente, alcanzando el máximo en E3 (0.872) y el mínimo en E8 (4.18) (Fig. 2 a). Por época, el valor de diversidad más alto se alcanzó en invierno de 1998, mientras que los más bajos se obtuvieron en otoño de 1997 y otoño de 1998. La riqueza específica y equidad también mostraron los valores 


\section{CUADRO 1}

Abundancia de las 11 especies de moluscos por localidades y época de muestreo. Abundance of 11 mollusk species

for each locality and season

\begin{tabular}{|c|c|c|c|c|c|c|c|c|c|c|c|c|}
\hline $\begin{array}{l}\text { Localidad } \\
\text { Época }\end{array}$ & M. ringens & H. oxyacantha & Cbrassicat & Cerythrostomus & Cregius & H.nigritus & H. princeps & C. gemmatus & P. princeps & V. caestus & C. fergusoni & Total \\
\hline El & 0 & 0 & 2 & 0 & 2 & 45 & 0 & I & 0 & 2 & 0 & 53 \\
\hline E2 & 0 & 4 & 0 & I & 3 & 16 & 0 & 0 & 1 & 0 & 0 & 24 \\
\hline E3 & 0 & 0 & 0 & 0 & 0 & 13 & 0 & 0 & 0 & 2 & 0 & 15 \\
\hline $\mathrm{E} 4$ & 0 & 0 & 0 & 0 & 3 & 180 & 0 & 0 & 0 & 2 & 0 & 185 \\
\hline E5 & 1 & 0 & 1 & 0 & 0 & 33 & 1 & 0 & 1 & 0 & 0 & 35 \\
\hline E6 & 9 & 0 & 0 & 1 & 9 & 188 & 0 & 1 & 0 & 4 & 1 & 212 \\
\hline E7 & 2 & 0 & 0 & 0 & 5 & 11 & 0 & 0 & 0 & 2 & 0 & 20 \\
\hline E8 & 0 & 0 & 2 & 6 & 24 & 203 & 0 & 0 & 1 & 7 & 1 & 245 \\
\hline E9 & 0 & 0 & 15 & 10 & 38 & 92 & 0 & 0 & 10 & 3 & 0 & 170 \\
\hline E10 & 0 & 0 & 0 & 0 & 3 & 119 & 0 & 0 & 0 & 2 & 0 & 124 \\
\hline Otoño 1997 & 5 & 2 & 0 & 6 & 15 & 270 & 0 & 1 & 3 & 4 & 0 & 305 \\
\hline Invierno 1998 & 4 & 0 & 9 & 0 & 18 & 80 & 0 & 0 & 9 & 2 & 2 & 124 \\
\hline Primavera 1998 & 0 & 0 & 7 & 2 & 14 & 154 & 1 & 0 & 0 & 3 & 0 & 182 \\
\hline Verano 1998 & 3 & 0 & 2 & 10 & 21 & 226 & 0 & 1 & 0 & 14 & 0 & 277 \\
\hline Otoño 1998 & 0 & 2 & 2 & 0 & 19 & 170 & 0 & 0 & 1 & 1 & 0 & 195 \\
\hline Biomasa & & & & & & & & & & & & \\
\hline Total (g) & 4028.3 & 165.7 & 2944.8 & 649.8 & 11608.3 & 77060.99 & 71.8 & 13.3 & 6454.3 & 31.829 & 698.6 & 106878.79 \\
\hline
\end{tabular}



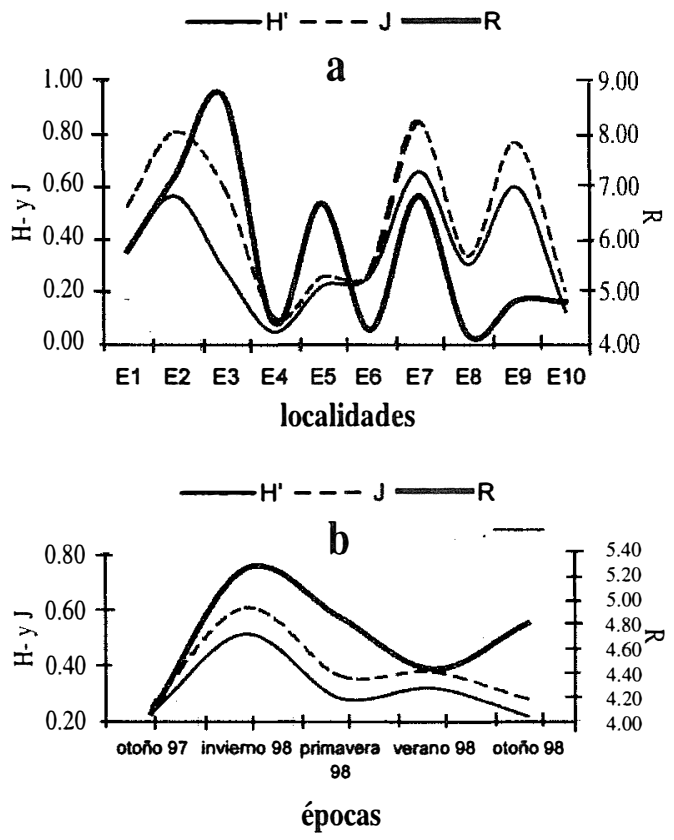

Fig. 2. Gráficos de índices univariantes: diversidad (H'), equitatividad (J) y riqueza (R). a) localidades de muestreo, b) época de muestreo.

Fig. 2. Univariant indeces graphics: diversity $\left(\mathrm{H}^{\prime}\right)$, evenness $(J)$ and specific richness (R). A) localities, b) season.

más altos en invierno de 1998, mientras que los valores más bajos se registraron en otoño de 1997. De esta forma los tres índices siguieron una variación casi paralela a lo largo de todo el período de muestreo (Fig. 2 b).

El análisis de ordenación efectuado con la matriz de localidades en las diferentes épocas no separó las diferentes muestras, obteniéndose una nube de puntos muy próximos entre sí. De hecho, el coeficiente de estrés fue alto (0.17), lo que indica una dudosa interpretación (Clarke 1993). En vista de estos resultados, se realizaron dos análisis de ordenación, uno entre las diferentes localidades y otro entre las diferentes épocas del período de muestreo. El análisis de las localidades, presentó un coeficiente de estrés bueno (0.11) y mostró dos grupos, uno constituído por las localidades El, E3, E4 E6 y E5, que se alejó de las anteriores, y el otro formado por E9, E8, E2, E7 y E10 (Fig. 3 a). El primer grupo de localidades se localizó en un rango de profundidad que varió

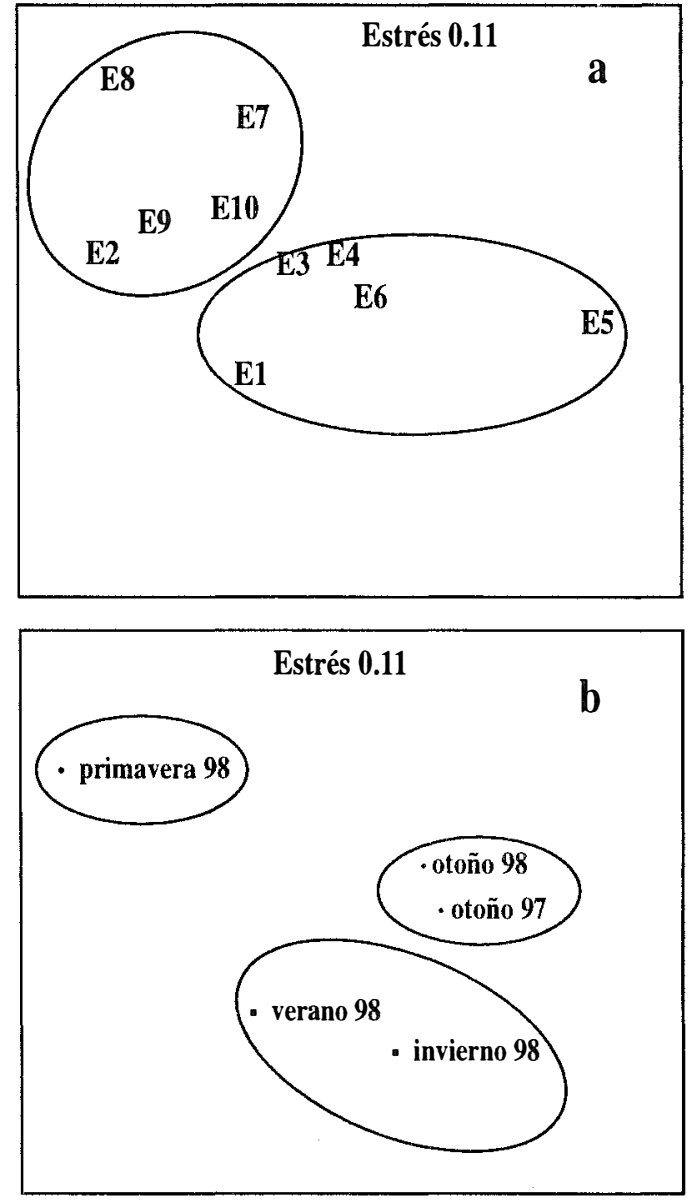

Fig. 3. Análisis de ordenación MDS. a) localidades de muestreo, b) época de muestreo.

Fig. 3. MDS ordination analysis. a) localities, b) season.

entre 5 y $13 \mathrm{~m}$, mientras que el segundo grupo se localizó a mayores profundidades ( $>15 \mathrm{~m})$, a excepción de E2 $(13 \mathrm{~m})$. El análisis de las épocas de muestreo (estrés= 0.02), mostró dos grupos, uno formado por las muestras de otoño de 1998 y de otoño de 1997, y el otro, constituído por las muestras de verano de 1998 e invierno de 1998; la muestra de primavera de 1998 se alejó de ambos grupos (Fig. 3 b).

Proporción de la captura langosta-moluscos: Con los datos de pesos registrados para langosta y moluscos durante este estudio, se estimó la proporción que representaron por estaciones del año, en total y por especies (Fig. 4 a, b). 


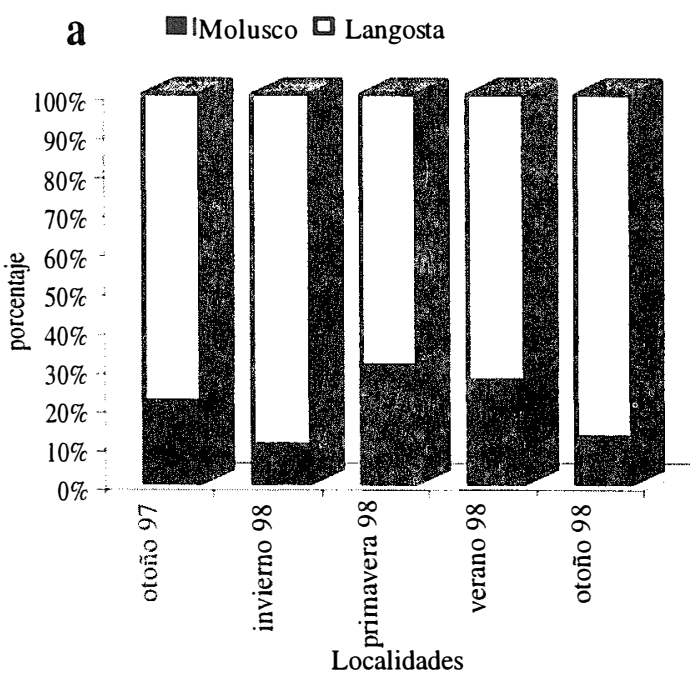

口primavera 98 verano 98 ๒otoño 97 ๒invierno 98 घotoño 98

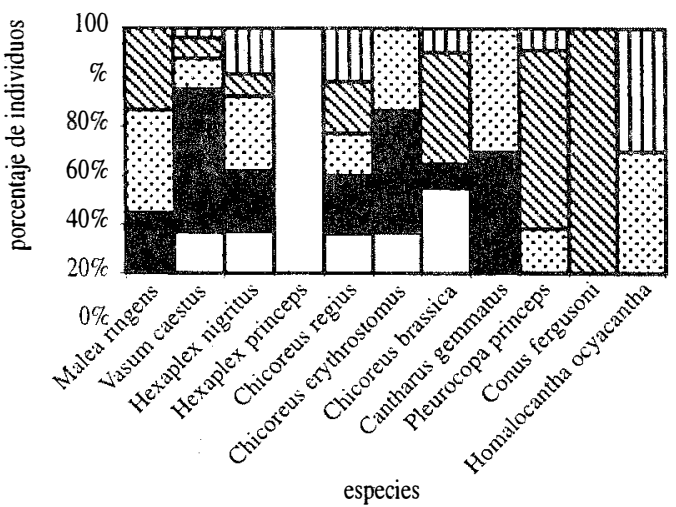

Fig. 4. Porcentaje de las capturas de langosta y molucos. a) porcentaje total de moluscos respecto al total de langosta, b) porcentaje de cada una de las especies de moluscos respecto al total de langosta.

Fig. 4. Percentage of lobster and mollusk captures. a) total mollusks percentage respect to total lobster, $b$ ) percentage of each mollusk species respect to total lobster.

En invierno de 1998 (enero-marzo) se obtuvo el menor porcentaje de moluscos $(10.20 \%)$ y el mayor de langosta $(89.8 \%)$, mientras que en primavera de 1998 (marzomayo) y verano de 1998 (junio-agosto) se registraron las mayores capturas de moluscos ( $30.82 \%$ y $27.03 \%$, respectivamente) y las menores de langosta $(69.18 \%$ y $72.97 \%$, respectivamente). Las redes se tendieron a una profundidad media de $19.2 \mathrm{~m}, 17.1 \mathrm{~m}$ y $7.75 \mathrm{~m}$, respectivamente.
Proporción de sexos y relaciones biométricas: La relación macho/hembra en estas especies fue de 1:1, salvo en C. erythrostomus y H. nigritus. La relación de sexos (394 machos: 506 hembras) en $H$. nigritus y en $C$. erythrostomus (13 machos: 5 hembras) se desvió significativamente de la relación 1:1 $(\mathrm{P}<0.001)$.

$H$. nigritus fue la única especie que presentó diferencias significativas de tamaño entre machos y hembras (Kruskal-Wallis, $\mathrm{P}<$ 0.001). Los machos presentaron una mayor frecuencia en la clase de $76 \mathrm{~mm}$, con una variación de tallas que osciló entre los 30 y 156 $\mathrm{mm}$, y las hembras alcanzaron la mayor frecuencia en la clase de $75 \mathrm{~mm}$, presentando tallas entre 36 y $106 \mathrm{~mm}$ (Fig. 5 a, b).

$V$. caestus mostró una distribución de tallas que osciló entre $58 \mathrm{~mm}$ y $94 \mathrm{~mm}$ (Fig. 5 c), alcanzando las mayores frecuencias en las clases comprendidas entre $76 \mathrm{~mm}$ y $88 \mathrm{~mm}$.

Dentro del género Chicoreus, los individuos de la especie $C$. regius alcanzaron la talla mayor, siendo las clases más abundantes, las comprendidas entre $101 \mathrm{~mm}$ y $115 \mathrm{~mm}$ (Fig. 5 d). C. brassica presentó la mayor frecuencia en la clase de $99 \mathrm{~mm}$, entre $79 \mathrm{~mm}$ y $129 \mathrm{~mm}$ (Fig. 5 e). C. erythrostomus resultó ser la especie más pequeña de este género, oscilando entre $52 \mathrm{~mm}$ y $102 \mathrm{~mm}$, con una frecuencia mayor en la clase de $87 \mathrm{~mm}$ (Fig. $5 \mathrm{f}$ ).

Las gráficas de relación talla-peso mostraron valores de pendiente próximos a 3 en $C$. brassica y $\mathrm{H}$. nigritus (Fig. 6), lo que indica que los organismos crecen y aumentan de peso proporcionalmente (Ricker, 1975). Sin embargo, en $C$. erythrostomus, $C$. regius y $V$. caestus los valores de la constante se alejaron de 3 , lo que indicaría un crecimiento alométrico.

Relación de abundancia y tallas con los parámetros físicos: $\mathrm{Al}$ estudiar los patrones de distribución del total de individuos se observó una mayor abundancia de éstos en profundidades comprendidas entre 14.4-21.6 m $(68.8 \%)$, aumentando en dirección norte (Fig. 7 a). Con respecto a la temperatura, se observó que el $53.7 \%$ de los ejemplares apareció en otoño de 1997 y verano de 1998, en un rango de temperatura comprendido entre $26.8^{\circ} \mathrm{C}$ y 

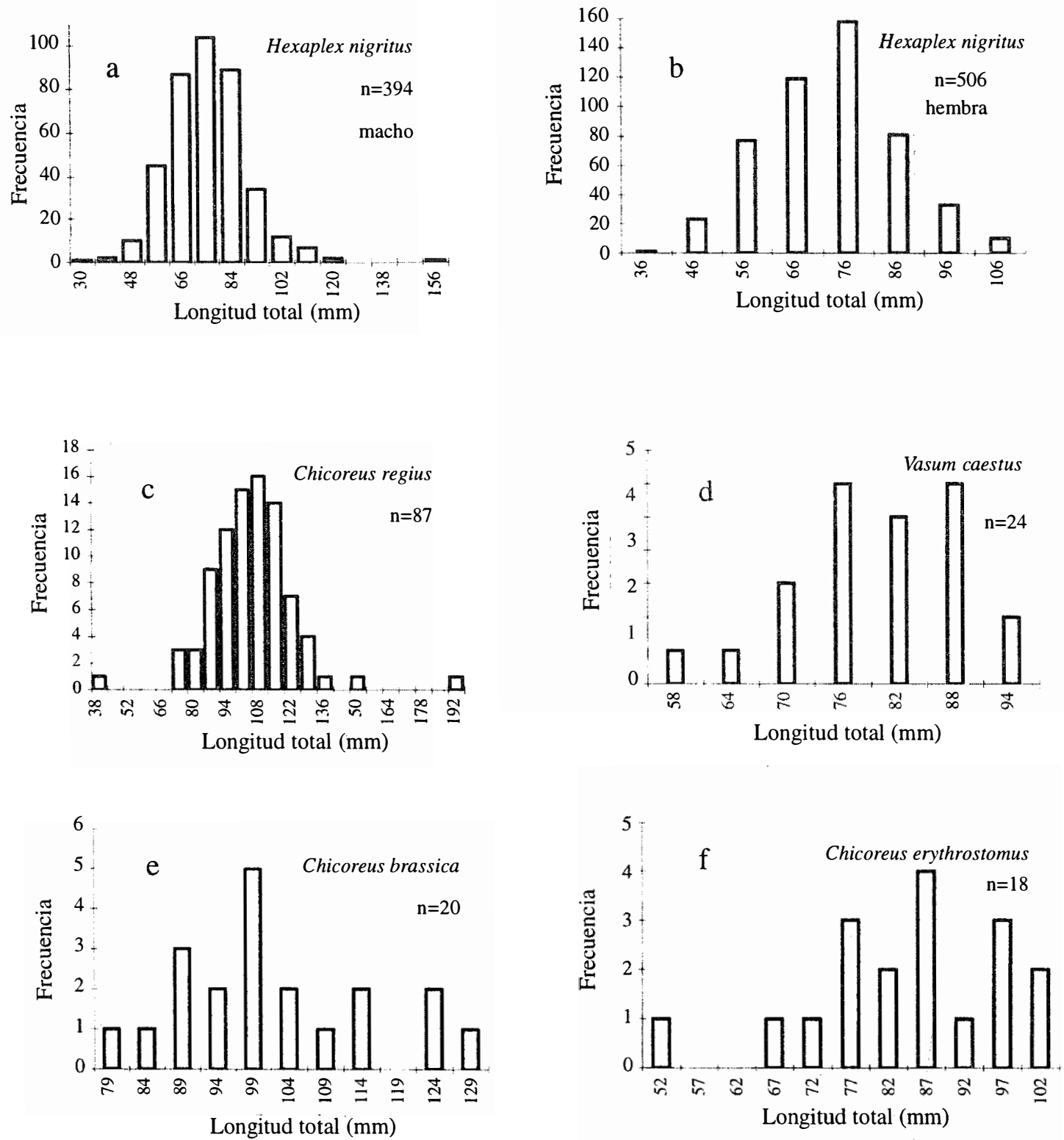

Fig. 5. Histogramas de frecuencias de tallas. a), b) H. nigritus, c) C. regius, d) V. caestus, e) C. brassica, f) C. erythrostomus. Fig. 5. Size frequency bars. a), b) H. nigritus, c) C. regius, d) V. caestus, e) C. brassica, f) C. erythrostomus. 

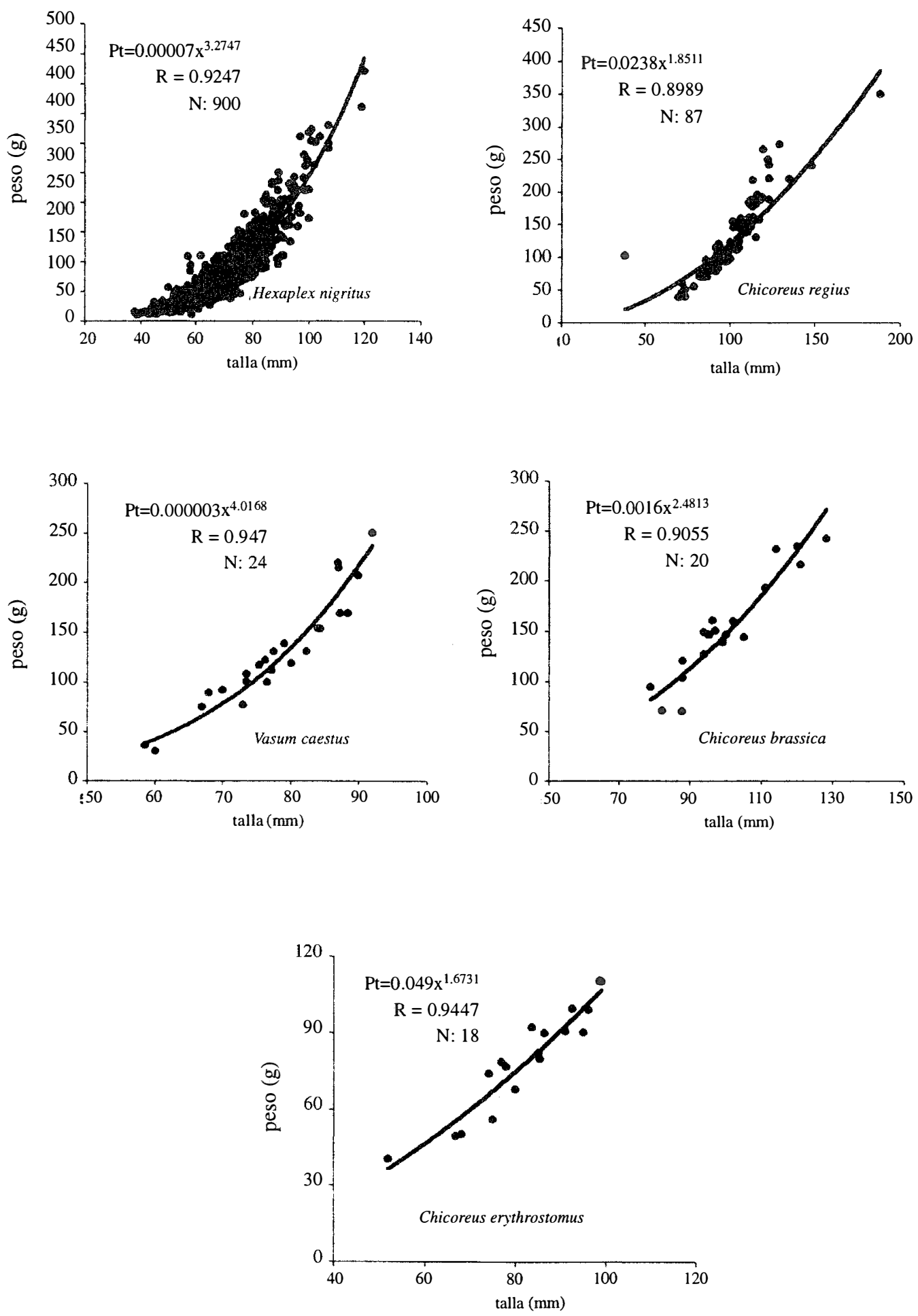

Fig. 6. Relación talla-peso para cada una de las especies más abundantes. Fig. 6. Weight-height ratio for dominant species. 


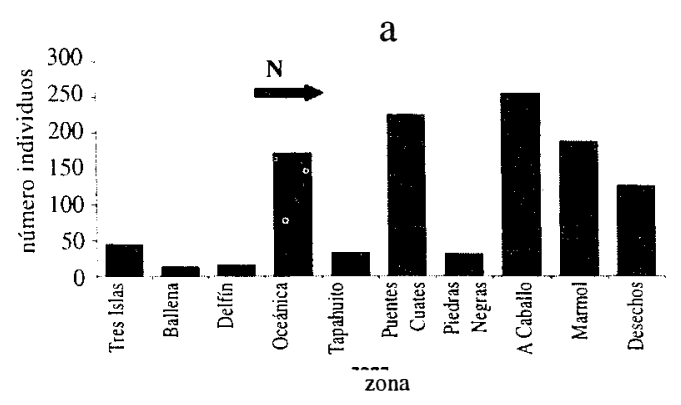

$\mathrm{b}$

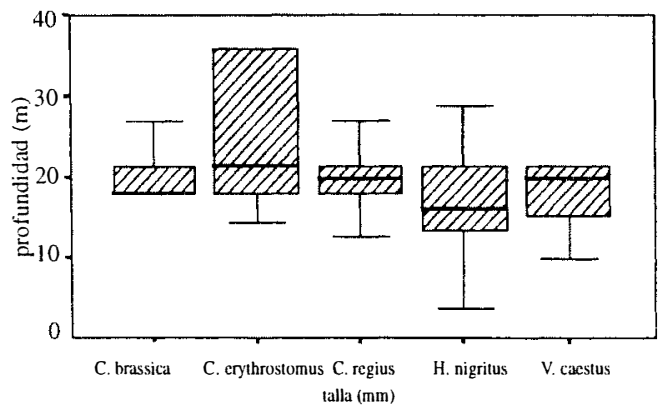

Fig. 7. Distribución de individuos: a) Variación del número total de individuos en las distintas zonas de estudio, b) Diagrama de cajas para la distribución de las especies más abundantes en relación con la profundidad.

Fig. 7. Individuals distribution: a) Variation of total individuals number at differente localities, b) Box plot for the dominant species distribution in relation with depth.

$30.8^{\circ} \mathrm{C}$. A pesar de estos patrones de distribución, no se obtuvieron correlaciones significativas entre el número de individuos y los parámetros profundidad ( $\mathrm{Ts}=0.262, \mathrm{P}>0.05$ ), $\mathrm{y}$ temperatura $(\mathrm{Ts}=-0.123, \mathrm{P}>0.05)$.

Las especies dominantes se distribuyeron de manera diferente en relación con la profundidad (Kruskal-Wallis, $\mathrm{P}<0.001$ ) (Fig. 7 b); $C$. erythrostomus apareció a mayores profundidades (>21 m), mientras que $H$. nigritus se encontró a profundidades menores (13-20 m). No se observaron diferencias significativas en la distribución de estas especies en relación con la temperatura (Kruskal-Wallis, $\mathrm{P}>0.05$ ).

La relación del tamaño con los parámetros ambientales solo presentó resultados estadísticos significativos en $H$. nigritus. Se presentaron diferencias significativas en la distribución de los tamaños con la profundidad y el área
(Kruskal-Wallis, $\mathrm{P}<0.001$ ). Sin embargo, al realizar un análisis de correlación de Spearman se obtuvo un coeficiente muy bajo entre talla y profundidad $(\mathrm{Ts}=-0.062, \mathrm{P}<0.05)$, es decir, no se ajustó a ningún patrón, aunque si se observó una ligera tendencia de disminución de la talla con la profundidad. Sin embargo, el análisis de correlación entre talla y temperatura mostró una correlación más alta (Ts = $0.308, \mathrm{P}<0.01$ ), encontrándose las tallas más grandes en los meses más fríos. Así mismo, la talla se correlacionó negativamente con el área de distribución (Ts $=-0.339, \mathrm{P}<0.01)$, disminuyendo el tamaño de los individuos a medida que nos desplazamos hacia el norte.

\section{DISCUSIÓN}

Índices univariantes y similitud entre localidades: El Niño, que causa temperaturas más altas de lo normal, particularmente en invierno (Quinn et al. 1978), ha sido considerado como uno de los factores de estrés más importantes que afectan a los ecosistemas marinos. Este fenómeno ha provocado reducciones en la caputra y altas mortalidades de macroalgas (Dayton \& Tegner 1984), muerte masiva en arrecifes de coral (Warwick et al. 1990) y mamíferos marinos (Suplee 1999), una disminución en la captura de especies como la sardina y un incremento de otras como el camarón (Rodríguez 1985). Además este fenómeno se ha asociado a la distribución de aves acuáticas (Ribic et al. 1997), al reclutamiento de larvas de moluscos (Moreno et al. 1998), o el reclutamiento de erizos de mar (Tegner \& Dayton 1991). Es posible que algunos patrones de variación de la diversidad, riqueza específica y abundancia observados en este trabajo sean consecuencia de este fenómeno.

En este estudio, la mayor abundancia y diversidad se encontró en otoño de 1997 e invierno de 1998, respectivamente, cuando se inició el fenómeno de El Niño, alcanzando su máximo desarrollo en invierno, con temperaturas de 3 a $4{ }^{\circ} \mathrm{C}$ por encima de lo normal. Kröncke $e t$ al. (1998) afirmaron la importancia e influencia 
de este tipo de sucesos climáticos en el aumento de la biomasa y biodiversidad de las comunidades bentónicas, puesto que en inviernos suaves la mortalidad, producción y reproducción no se reducen tanto como en inviernos más fríos, comenzando la productividad primaria más pronto. Asimismo, Beukema et al. (1996) han indicado la importancia que juegan los factores climáticos en la estructuración del bentos. Beukema (1992) y Cushing \& Dickson (1976) encontraron que después de inviernos templados se producía un incremento del número de especies y un aumento de la biomasa, resultados que concuerdan con los de este trabajo. Moreno et al. (1993) y Moreno et al. (1998) estudiaron la influencia de el fenómeno de El Niño y La Niña en el reclutamiento de moluscos murícidos, indicando que se producía un descenso de éste durante estos fenómenos. Nosotros pudimos observar un descenso en las capturas de los murícidos, en especial en $H$. nigritus, sin embargo, no se puede asegurar que esto sea debido a la influencia del fenómeno de El Niño, y es necesario hacer estudios a larga plazo con el objeto de conocer realmente los efectos de este fenómeno en las poblaciones de esta especie.

Se apreció un incremento del número de moluscos en dirección norte, debido al tipo de sustrato del fondo, encontrándose una mayor extensión de zona rocosa en esa dirección (Melchor-Aragón 1998), que es el hábitat preferencial de las especies objeto de estudio. Además del tipo de sustrato, otros factores como la abundancia y disponibilidad de alimento, sedimentación y corrientes, son factores que pueden determinar la distribución de estos individuos (Turrubiates-Morales \& Castro-Ortiz 1992, Cupul-Magaña \& Torres-Moye 1996).

La riqueza específica siguió un patrón de variación similar a la diversidad y equidad. Los valores de riqueza fueron bajos en relación a los observados en otros trabajos de fauna asociada a la pesca artesanal de langosta (Ayón-Parente et al. 1997, Pérez-González et al. 1997). Esto puede explicarse en función del arte de pesca utilizado. En este estudio todos los organismos recolectados fueron gasterópo- dos, mayormente epifaúnicos, mientras que gran número de bivalvos son semiinfaúnicos o infaúnicos, por lo que la probabilidad de ser capturados por la red es mayor para los gasterópodos que para los bivalvos. Por otro lado, la mayoría de las familias de moluscos epifaúnicos tienen tamaños inferiores a la luz de malla de la red $(8.75 \mathrm{~cm}-15 \mathrm{~cm})$.

La separación obtenida en el análisis de ordenación de las localidades pudo estar reflejando variaciones de las características locales, que determinaron una composición específica diferente. Las localidades menos profundas presentaron temperaturas más elevadas, mientras que las más profundas presentaron temperaturas inferiores $\left(0.5-2^{\circ} \mathrm{C}\right.$ menor que la temperatura superficial). Asimismo, el tipo de fondo varió, predominando la arena en las localides menos profundas, y arena con sustrato rocoso en las más profundas. Aunque los moluscos aparecen bajo un amplio rango de condiciones ambientales (Hendrickx et al. 1984b), el tipo de sustrato es un factor determinante de la distribución de estos organismos, principalmente en la zona infralitoral (Currás \& Mora 1991, Bachelet \& Dauvin 1993, Olabarria et al. 1998). También, la disponibilidad de alimento, la profundidad y latitud que afectan en el gradiente de disponibilidad de alimento, y la velocidad de la corriente de agua, son los factores que provocan la distribución de las diferentes especies bentónicas (Pearson \& Rosenberg 1987).

Biometría y relación con parámetros físicos: La relación talla-peso mostró valores de la pendiente próximos a 3 en $C$. brassica y $H$. nigritus, lo que indica según Ricker (1975) que los organismos crecen y aumentan de peso proporcionalmente. Sin embargo, en C. erythrostomus, $C$. regius y $V$. caestus los valores de la constante se alejaron de 3, lo que indicaría un crecimiento alométrico. El crecimiento alométrico en varias especies de moluscos bivalvos y cefalópodos ha sido observado por varios autores (Olivier \& Capitoli 1980, Arizpe \& Félix 1986, Hanlon \& Forshyte 1987, Castro-Ortiz \& García-Domínguez 1993).

En el caso de $H$. nigritus se obtuvo una correlación negativa con la temperatura, observándose 
las tallas y pesos más grandes en los meses más fríos. Esto puede ser debido a una mayor disponibilidad de alimento en esta época, puesto que son organismos depredadores de bivalvos fundamentalmente, y es en esta época cuando estas especies de bivalvos están sometidas a menor presión pesquera. Sin embargo, estos resultados no parecen estar de acuerdo con los de otros autores, que indican que los moluscos, principalmente bivalvos, presentan tallas más grandes en períodos más cálidos (Arizpe \& Félix 1986, Jones et al. 1990, Arnold et al. 1991, Castro 1992, Castro-Ortiz et al. 1992, Castro-Ortiz \& García-Domínguez 1993). Sin embargo, hay que considerar que en este invierno la temperatura aumentó $3-4{ }^{\circ} \mathrm{C}$ con respecto a años anteriores, lo que pudo originar un mayor crecimiento de estos organismos. La ligera tendencia a disminuir la talla con la profundidad en $H$. nigritus, también ha sido observada por otros autores en estudios de crecimiento de moluscos (Arnold et al. 1991, Castro-Ortiz et al. 1992).

Los resultados mostraron una alta abundancia de $H$. nigritus respecto a las demás especies. Esta especie de un importante valor comercial (Poutiers 1995), podría ser objeto de una nueva pesquería. Los resultados sugieren que el esfuerzo pesquero se podría enfocar hacia las zonas de mayor densidad de individuos (norte de la Bahía de Mazatlán, entre 13 y $20 \mathrm{~m}$ de profundidad) en la época de otoño, cuando se encontró una mayor densidad. Sin embargo, hay que tener en cuenta que este estudio se llevó a cabo durante un año Niño, de forma que las condiciones climatológicas-oceanográficas respondieron a un patrón diferente del normal. Son necesarios estudios posteriores, de varios ciclos anuales, para determinar el comportamiento de las poblaciones de esta especie, principalmente en relación con patrones de distribución espacio-temporal, relaciones entre reproducción y patrones de movimiento con cambios estacionales de temperatura, salinidad, corrientes y otros factores físicos. Asimismo, se necesitan estudios de épocas de reproducción, tasa de fecundidad y relación entre época de reproducción y patrones de migración.

\section{AGRADECIMIENTOS}

La autora agradece de manera especial a Raúl Pérez González por su apoyo y su total disposición a prestar ayuda durante todo este tiempo. También a los pescadores de las Cooperativas Punta Tiburón y José María Canizalez, a Luis Miguel Valadez Manzano, Francisco Arzola González, Manuel Ayón Parente, Rolando Quintero Montoya y Gilberto Robles Cruz, por su ayuda en la recolección de las muestras. Asimismo, la autora agradece las valiosas aportaciones de los revisores del trabajo, que contribuyeron a una notable mejora del mismo.

\section{RESUMEN}

En este trabajo se estudió la fauna malacológica asociada a la pesca de la langosta en aguas del Pacífico Tropical durante 1997-1998. Se recogieron un total de 1081 individuos pertenecientes a 11 especies. Las mayores capturas se obtuvieron en otoño de 1997 (28.16\%) y en verano de 1998 (25.58\%). Las especies dominantes fueron $H$. nigritus $(83.10 \%), C$. regius $(8.04 \%), V$. caestus $(2.12 \%)$, C. brassica (1.85\%) y C. erythrostomus $(1.75 \%)$, las cuales tienen importancia comercial. Se calculó la relación talla-peso, histogramas de frecuencia de tallas, y la relación de sexos para las especies dominantes. La relación de sexos fue de $1: 1$, salvo en $C$. erythrostomus y $H$. nigritus (2 machos: 1 hembra); en $C$. regius, $C$. erythrostomus y $V$. caestus el crecimiento fue alométrico mientras que en $H$. nigritus y $C$. brassica fue isométrico. Solamente, los machos de $H$. nigritus fueron más grandes que las hembras de la misma especie. Se encontraron diferencias significativas en la distribución de las especies respecto a la profundidad; C. erythrostomus apareció a mayores profundidades mientras que $H$. nigritus estuvo en zonas más someras. El tamaño de $H$. nigritus varió con la profundidad, temperatura y localidad.

\section{REFERENCIAS}

Arizpe, C.O. \& P. Félix. 1986. Crecimiento de Pinna rugosa (Sowerby, 1835) en la Bahía de la Paz, México. An. Inst. Cienc. Mar y Limnol. Univ. Nal. Autón. México 13: 167-172.

Arnold, W.S., D.C. Merelli, T.M. Bert, D.S. Jones \& I.R. Quitmyer.1991. Habitat-specific growth of hard clams Mercenaria mercenaria from the Indian River, Florida. J. Exp. Mar. Ecol. 147: 245-265. 
Ayón, M., M.E. Hendrickx \& R. Pérez-González. 1997. Fauna carcinológica asociada a la pesca de la langosta (Panulirus spp.), durante 1995-1996, en el sur de Sinaloa. Informe Técnico Abril 1994- Marzo 1997, Proyecto CONACYT 0948-N9111, Mazatlán. 326 p.

Bachelet,G. \& J.C. Dauvin. 1993. Distribution quantitative de la macrofaune benthique des sables intertidaux du bassin d'Arcachon. Oceanol. Act. 16: 83-97.

Beukema, J.J. 1992. Expected changes in the Wadden Sea benthos in a warmer world: lessons from periods with mild winters. Neth. J. Sea Res. 30: 73-79.

Beukema, J.J., K. Essink \& H. Michaelis. 1996. The geographic scale of synchronized fluctuation patterns in zoobenthic populations as a key to underlying factors: climatic or man-induced. ICES J. Mar. Sci. 53: 964-971.

Bray, R.T. \& J.T. Curtis. 1957. An ordination of the upland forest communities of southern Wisconsin. Ecol. Monogr. 27: 325-349.

Castro, O.J.L. 1992. Crecimiento de la almeja chocolata Megapitaria squalida (Sowerby, 1835) en Bahía Concepción, Baja California Sur, México. Inv. Mar. CICIMAR 1: 1-7.

Castro-Ortiz, J.L. \& F. García-Domínguez. 1993. Crecimiento de Chione californiensis (Broderip, 1635) (Veneridae) en la Ensenada de la Paz, Baja California Sur, México. Inv. Mar. CICIMAR 8: 45-52.

Castro-Ortiz, J.L., A. Tripp-Quezada \& B. Anguas-Vélez. 1992. Crecimiento de la almeja chocolata Megapitaria squalida (Sowerby, 1835) en Bahía Concepción, Baja California Sur, México. Inv. Mar. CICIMAR 7 $1-7$.

Clarke, K.R. 1993. Non-parametric multivariate analyses of changes in community structure. Austr. J. Ecol 135: $109-122$

Cupul-Magaña, F.G. \& G. Torres-Moye. 1996. Age and growth of Astraea undosa Wood (Mollusca: Gastropoda) in Baja California, Mexico. Bull. Mar. Sci. 59: 490-497.

Currás, A. \& J. Mora. 1991. Comunidades bentónicas de la Ría del Eo (Galicia-Asturias, NW España). Cah. Biol. Mar. 32: 57-81.

Cushing, D. H. \& R.R. Dickson. 1976. The biological response in the sea to climatic changes. Adv. Mar. Biol. 14: 2-122.

Dajoz, R. 1971. Précis d'Écologie. Dunod, Paris. 273 p.
Dayton, P.K \& M.J. Tegner.1984. Catastrophic storms, El Niño, and patch stability in a Southern Califormia kelp community. Science 224: 283-285.

Field, J.G, K.R. Clarke \& M. Warwick. 1982. A practical strategy for analysing multispecies distribution patterns. Mar. Ecol. Prog. Ser. 8: 37-52.

Flores-Campaña, L.M., R. Pérez-González \& A. NuñezPasten, 1993. La pesquería de las langostas Panulirus inflatus (Bouvier) y $P$. gracilis Streets en la costa sureste del Golfo de California. Memorias del Taller Binacional sobre la utilización de refugios artificiales en las pesquerías de las langosta: sus implicaciones en la dinámica y manejo del recurso. Secretaría de Pesca-INP, México/Centro de Investigaciones Pesqueras de Cuba . Isla Mujeres, Quintana Roo, México. $121 \mathrm{p}$.

García, E. 1973. Modificaciones al sistema de clasificación climática de Köpen (adaptación a condiciones de la República Mexicana). Inst. Geogr. Univ. Nal. Autón, México. 246 p.

Hanlon, R. \& L. Forshyte. 1987. Growth, p. 135-156. In Boyle, E. (ed.). Cephalopod life cycles II. Academic Press, Londres.

Hendrickx, M.E. 1985. Diversidad de los macroinvertebrados bentónicos acompañantes del camarón en el área del Golfo de California y su importancia como recurso potencia, p. 95-148. In Yáñez-Arancibia, M. (ed.). Recursos pesqueros potenciales de México: $\mathrm{La}$ pesca acompañante del camarón. Prog. UNAM, México D.F.

Hendrickx, M.E. 1990. Nuevas localidades de colecta de Raninoides benedicti Rathbun, 1935 (Brachyura: Raninidae), con notas ecológicas y observación de una alometría de crecimiento de las quelas. Rev. Biol. Trop. 38: 343-345

Hendrickx, M.E., A.M. Van der Heiden \& A. ToledanoGranados. 1984a. Resultados de las campañas SIPCO (Sur de Sinaloa, México) a bordo del B/O "El Puma". Hidrología y composición de las capturas efectuadas en los arrastres. An. Inst. Cienc. Mar y Limnol. Univ. Nal. Autón. México 11: 107-122.

Hendrickx, M.E., A.M. van der Heiden \& A. ToledanoGranados. 1984b. Results of the SIPCO cruises (Southem Sinaloa, Mexico) aboard the B/O "El Puma". Abundance and distribution of commercially exploitable mollusks. Rev. Biol. Trop. 32: 69-75.

Hendrickx, M.E., R. Pérez-González \& L.M. Flores-Campaña. 1992. Identificación y colectas del cangrejo araña Maiopsis panamensis Faxon, 1895 (Brachyu- 
ra: Majidae) en el Pacífico Este Tropical. Rev. Cien. Mar. UAS 12: 31-34.

Hendrickx, M.E., R. Pérez-González, L.M. Flores-Campaña \& M. Ayón-Parente. 1997. Nuevas capturas de dos especies raras de cangrejos braquiuros (Crustacea: Brachyura) para la costa del Pacífico Este Tropical. Rev. Cien. Mar. UAS 15: 51-54.

Jones, S.D., I.R. Quitmyer, W.S. Arnold \& C. Marelli. 1990. Annual shell banding, age, and growth rate of hard clam (Mercenaria spp.) from Florida. J. Shellfish. Res. 9: 215-225.

Juárez-Rosales, J. 1995. Eficiencia y desarrollo tecnológico de las redes de enmalle para la pesca de langostas en el sur de Sinaloa, México. Oceanología 4: 85-93.

Kröncke, I., J.W. Dippner, H. Heyen \& B. Zeiss. 1998. Long-terms changes in macrofaunal communities off Norderney (East Frisia, Germany) in relation to climate variability. Mar. Ecol. Prog. Ser. 167: 25-36.

Margalef, A.R. 1974. Ecología. Omega, Barcelona. 915 p.

Melchor-Aragón, J.M. 1998. La pesquería del ostión de roca (Crassostrea iridescens) en San Ignacio Sinaloa, México: propuesta de un modelo de simulación para su manejo. Tesis de Maestría, Facultad de Ciencias del Mar, Universidad Autónoma de Sinaloa, Mazatlán, Sinaloa, México.

Moreno, C.A., G. Asencio \& S. Ibañez. 1993. Patrones de asentamiento de (Bruguière) (Mollusca: Muricidae) en la zona intermareal rocosa de Valdivia, Chile. Rev. Chil. Hist. Nat. 66: 93-101.

Moreno, C.A., G. Asencio, W.E. Duarte \& V. Marín. 1998. Settlement of the muricid Concholepas concholepas and its relationship with El Niño and coastal upwellings in southern Chile. Mar. Ecol. Prog. Ser. 167: 171-175.

Olabarria, C., V. Urgorri \& J.S. Troncoso. 1998. An analysis of the community structure of subtidal and intertidal benthic mollusk of the Inlet of Baño (Ría de Ferrol) (northwestern Spain). Am. Malacol. Bull. 14: 103-120.

Olivier, S.R. \& R. Capitoli. 1980. Edad y crecimiento en Chlamys tehuelcha (D’Orbigny) (Mollusca, Pelecypoda, Pectinidae) del Golfo San Matías (PCIA, do Río Negro, Argentina). An. Inst. Cienc. Mar y Limnol. Univ. Nal. Autón. México 7: 129-140.

Pearson, T.H. \& R. Rosenberg. 1987. Feast and famine: structuring factors in marine benthic communities,
373-395 p. In Gee, J.E. \& Giller, V. (eds.) Organization of communities, past and present. Blackwell Scientific Publications, Oxford.

Pérez-González, R., L.M. Flores-Campaña, M.I. Borrego \& L.M. Valadez Manzano. 1994. Fauna asociada a la pesca de la langosa durante la época fría, en el sur de Sinaloa. Rev. Cien. Mar. UAS 13: 21-26.

Pérez-González, R., S.A. López-Machado, L.M. FloresCampaña \& R. Salazar-Carvajal.1997. Composición de la fauna incidental en las capturas de la pesca de la langosta Panulirus sp. en el sureste del golfo de California. Informe Técnico Abril 1994- Marzo 1997, Proyecto CONACYT 0948-N9111, Mazatlán. 326 p.

Pielou, E.C. 1966. The measurement of diversity in different types of biological collections. J. Theoret. Biol. 13: 131-144.

Poutiers, J.M. 1995. Bivalvos, p 100-221.Gasterópodos, p. 224-297. In Fisher, W., Krupp, F., Schneider, W., Sommer, C., Carpenter, K.E. \& Niem, V.H. (eds.). Guia FAO para la identificación de especies para fines de pesca. Pacífico Centro oriental, vol I. Plantas e Invertebrados. FAO, Roma.

Quinn, W.H, D.O. Zopf, K.S. Short \& R.T.W. Kuo Yang.1978. Historical trends and statistics of the southern oscilation, $\mathrm{El} \mathrm{Niño,} \mathrm{and} \mathrm{Indonesian}$ droughts. Fish. Bull. U.S. 76: 663-678.

Ribic, C.A, G.A. Davis \& L.B. Spear. 1997. Scale-related seabird-environmental relationships in Pacific equatorial waters, with reference to El Niño-Southern Oscillation events. Mar. Ecol. Prog. Ser. 156: 183-203.

Ricker, W.E. 1975. Computation ańd interpretation of biological statistics of fish populations. Bull. Fish. Res. Board. Can. 191: 1-382.

Rodríguez, G. 1985. El Niño. Notas sobre el efecto en las comunidades nectónicas y marinas. Rev. Cien.' Mar. UAS 7: $27-30$

Rodríguez de la Cruz, M.C. 1981. Aspectos pesqueros del camarón de alta mar en el Pacífico Mexicano. Ciencia Pesquera. Inst. Nal. Pesca. Dpto. Pesca, México 1: 43-55.

Shannon, E.C. \& W. Weaver. 1963. The mathematical theory of communication. Illinois University Press, Urbana, Illinois. 119 p.

Snedecor, G.W. \& W.G. Cochran. 1980. Stadistical methods. Iowa State University Press, Ames, Iowa. 507 p. 
Sokal, R.R. \& Rohlf, J. 1979. Biometry. Blume, Madrid. $389 \mathrm{pp}$.

Suplee, C. 1999. El Niño/La Niña. National Geographic 4: 74-95.

Tegner, M.J. \& P.K. Dayton. 1991. Sea urchins, El Niños, and the long term stability of Southern California kelp forest communities. Mar. Ecol. Prog. Ser. 77: 49-63.

Turrubiates-Morales, R. \& J.L. Castro-Ortiz. 1992. Growth of Haliotis fulgens in Bahía Tortugas, Baja California Sur, Mexico, p. 10-15. In Guzmán del Próo, Tegner \& Shepherd (eds.). Abalone of the world: Fisheries and culture (supplementary papers). Fisheries Research Papers, Department of Fisheries, South Australia. van der Heiden, A. \& M. E. Hendrickx. 1982. Inventario de la Fauna Marina y Costera del Sur de Sinaloa. Segundo Informe de Avance. Estación Mazatlán. Inst. Cienc. Mar y Limnol. UNAM. 135 p.

van der Heiden, A. \& H. G. Plasencia-González. 1997. Primeros registros de Syacium longidorsale Murakami y Amaoka, 1992 (lenguado de aleta larga: Pleuronectiformes: Paralichthyidae) y de Brotula ordwayi Hildebrand y Barton, 1949 (congrio pintado: Ophidiiformes: Ophiidae) para la costa Pacífica de México. Vertebrata Mexicana 4: 5-8.

Warwick R.M, K.R. Clarke \& L. Suharsono. 1990. A statistical analysis of coral community responses to the 1982-3 El Niño in the Thousand Islands, Indonesia. Coral Reefs 8: 171-179. 